\title{
THE RELATION OF THE SERUM ANTISTREPTOLYSIN TITER TO THE EXACERBATION IN CHRONIC GLOMERULONEPHRITIS
}

\author{
By DAVID P. EARLE, JR., DAVID SEEGAL, JOHN D. LYTTLE, EMILY N. LOEB, \\ AND ELIZABETH L. JOST \\ (From the Research Service, First Division, Welfare Hospital, Department of Hospitals; \\ Presbyterian Hospital; Babies Hospital; and the Departments of Medicine and Pediatrics, \\ College of Physicians and Surgeons, Columbia University, New York City)
}

(Received for publication March 6, 1942)

In the present study, the relation of the serum antistreptolysin titer to the exacerbation in chronic glomerulonephritis has been analysed. In a previous study (1), an exacerbation in chronic glomerulonephritis was defined arbitrarily as an abrupt and marked increase in the degree of hematuria. It was pointed out that slight variations in the urinary output of erythrocytes perhaps do not signify the presence of an exacerbation. Exacerbations were frequently associated with some impairment of renal function, generally transient in character. Twenty-eight such episodes were reported in an analysis of 68 cases of chronic glomerulonephritis. The present study is based on this material, with the addition of 5 exacerbations that have been observed since the completion of the earlier study. Two of these exacerbations occurred in a patient (Case XI, PA) previously reported (1), while the other 3 were observed in 2 of 13 new cases studied.

The 33 exacerbations in chronic glomerulonephritis have been observed in 15 of 81 cases, studied over periods of 4 months to 8 years. Each exacerbation was preceded by an upper respiratory tract infection. Group A hemolytic streptococci were recovered by throat culture from 17 of these instances. Adequately studied rises in antistreptolysin titer, as previously defined (2), were associated with 20 of these exacerbations. Definite rises in titer were associated with 4 additional exacerbations, but the data were not sufficient to determine the maximum titer of the rise and therefore not considered in this study.

Six exacerbations were not associated with rises in antistreptolysin titer. In each of these instances determinations were done with sufficient frequency to rule out the possibility of a rise. Group A hemolytic streptococci were recovered by throat culture in one of these instances, hemolytic streptococci whose group was not determined in another, while the other 4 instances followed infections not proven to be due to the hemolytic streptococcus (head colds in 2, pneumococcus type XXIII otitis media in 1 , and lobar pneumonia, causative organism unknown, in 1).

Finally, in 3 instances of exacerbation, determinations were not done with sufficient frequency to discover the presence of a possible brief rise in antistreptolysin titer. Hemolytic streptococci were not recovered by throat culture from any of these 3 instances, the associated infections being head colds, "grippe," and pneumococcus type II ethmoiditis. These instances are not included in the present study.

During the 4 months to 8 year period of observation on these 81 cases of chronic glomerulonephritis, 76 adequately studied rises in antistreptolysin titer were observed in which urinalyses yielded sufficient data to determine the presence or absence of associated exacerbation in chronic glomerulonephritis. The present study does not include 9 of the 85 adequately studied rises in antistreptolysin titer ${ }^{1}$ reported in the preceding paper (2).

In summary, the data from which this paper is compiled were obtained from 81 cases of chronic glomerulonephritis in which 76 rises in antistreptolysin titer and 26 exacerbations in chronic glo-

\footnotetext{
1 These rises in titer were eliminated from this study for the following reasons: (1) Although rises in titer were associated with sharp increases in the degree of hematuria, the numbers of erythrocytes excreted in the urine during control periods were so variable that it was impossible to determine whether an exacerbation was present or not (4 instances); (2) Hematuria followed an intravenous injection of hemolytic streptococcus nucleoprotein (1 instance); (3) The rise in titer occurred too close to the time of death to permit the development of an exacerbation ( 3 instances); and (4) The rise in titer followed an unobserved episode that may or may not have been an exacerbation ( 1 instance).
} 
merulonephritis were both adequately studied. Thus, a correlation or lack of correlation between the two reactions could be determined.

RELATION OF THE MAGNITUDE OF ANTISTREPTOLYSIN TITER RESPONSE TO THE OCCURRENCE OF EXACERBATION IN CHRONIC GLOMERULONEPHRITIS

The 76 rises in antistreptolysin titer in this series were analysed for the presence or absence of exacerbation in chronic glomerulonephritis. It was noted that only rises that attained a maximum titer of 144 units or more were associated with exacerbations. Further, 13 of the 15 rises that attained a maximum titer of 333 units or more were associated with exacerbations. These points are graphically presented in Figure 1 where it may be seen that the higher the maximum titer of the rise, the more frequent is the occurrence of exacerbation. This figure also shows a similar although not so striking correlation between the magnitude of the rise above the base-line values and the presence or absence of exacerbation.

It should be emphasized once more that $6 \mathrm{ex}$ acerbations were not associated with rises in antistreptolysin titer. However, in only one of these

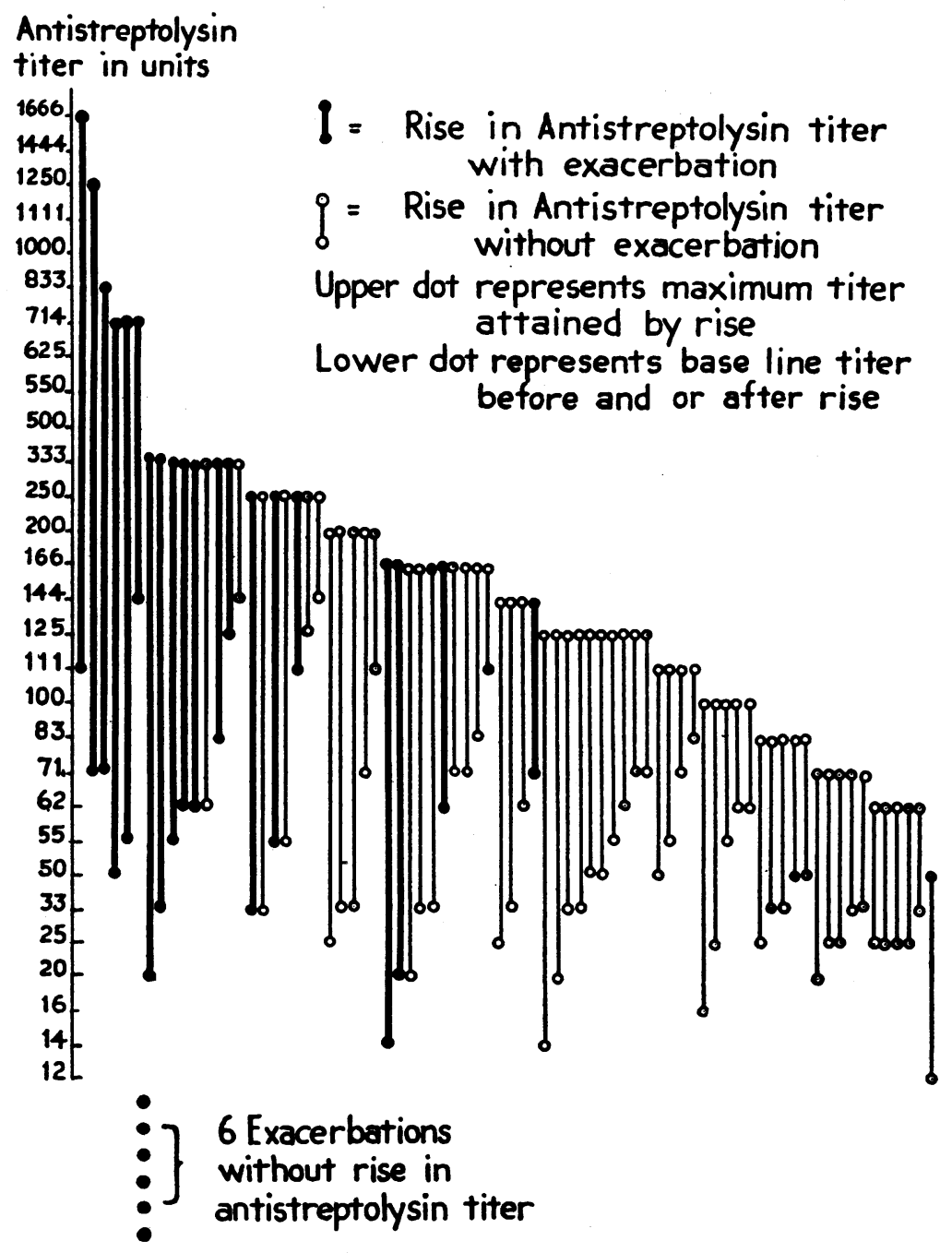

Fig. 1. Relation of the Maximum Titer Attained by 76 Rises in AntiStreptolysin and the MAgnitude of THE Rises to the Occurrence of Exacerbation in Chronic Glomerulonephritis 
instances was the preceding infection proven to be due to Group A hemolytic streptococci.

As previously indicated (1), the preponderance of observed exacerbations in chronic glomerulonephritis occurred in children. Furthermore, it has been shown (2) that rises in antistreptolysin titer tended to be greater among the younger patients suffering from chronic glomerulonephritis than among the adults. Thus, both exacerbations and large rises in antistreptolysin titer were uncommon among adults. However, 3 of the 6 rises attaining a maximum titer of 250 units or more that were observed in patients 20 years of age or older were associated with exacerbations. These relations are shown in Figure 2. It seems likely, therefore, that there is a more direct correlation

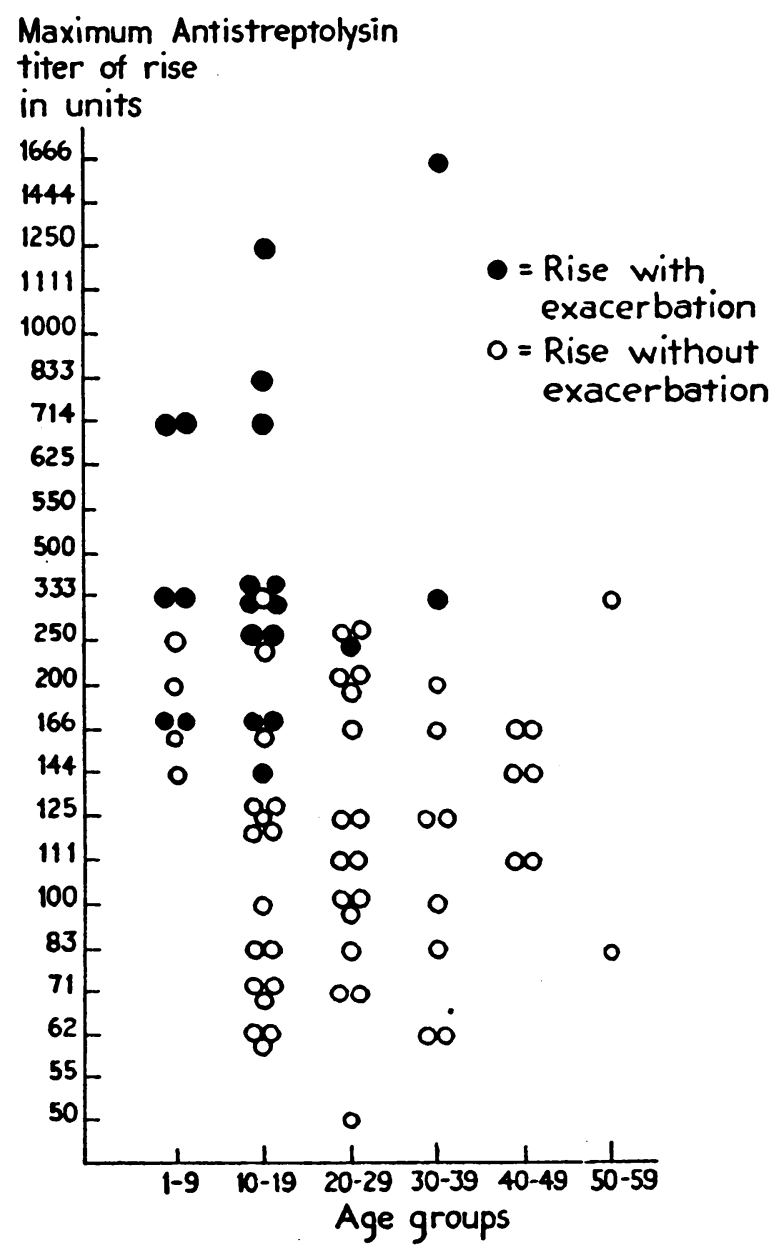

Fig. 2. Relation of the Occurrence of Exacerbation in Chronic Glomerulonephritis to the Maxima of Rises in Antistreptolysin Titer and to Age between the magnitude of the antistreptolysin titer response and the incidence of exacerbations than between age and the incidence of exacerbations.

In the preceding paper (2) it was pointed out that the antistreptolysin titer response was, in general, greater among patients with chronic glomerulonephritis without nephrotic edema than among patients with edema. Thus, 19 of the 50 rises observed among patients without the nephrotic element attained a maximum titer of 250 units or more, while only 6 of the 35 rises found in edematous patients reached similar levels. However, the incidence of exacerbations associated with rises of this magnitude was approximately the same in patients without and with edema, 13 of 17 and 3 of 5 respectively (disregarding the several rises associated with equivocal exacerbations). Thus, there appears to be a more direct correlation between the magnitude of the antistreptolysin titer response and the incidence of exacerbations, than between the status of the nephritis (with or without nephrotic edema) and the occurrence of exacerbations.

RELATION OF THE ONSET OF ANTISTREPTOLYSIN TITER RISE TO THE ONSET OF EXACERBATION IN CHRONIC GLOMERULONEPHRITIS

In 8 instances of exacerbation, antistreptolysin titer determinations were done at intervals that permitted conclusions as to whether the exacerbation began before, during, or after the onset of the rise in titer. These 8 instances are shown in Figure 3, where the onset of infection is represented by the heavy vertical line. Control antistreptolysin titer values are shown to the left of this line, while the rises in titer are on the right. The onset of hematuria in each instance is indicated by an arrow.

It may be noted in Figure 3 that the exacerbation occurred before the rise in antistreptolysin titer was apparent in 7 of these 8 instances. This is not surprising in view of the brief latent period between the preceding infection and the onset of the exacerbation in chronic glomerulonephritis (3). In addition, each of the 20 exacerbations associated with adequately studied rises in antistreptolysin titer occurred well before the peak of the rise was reached. 


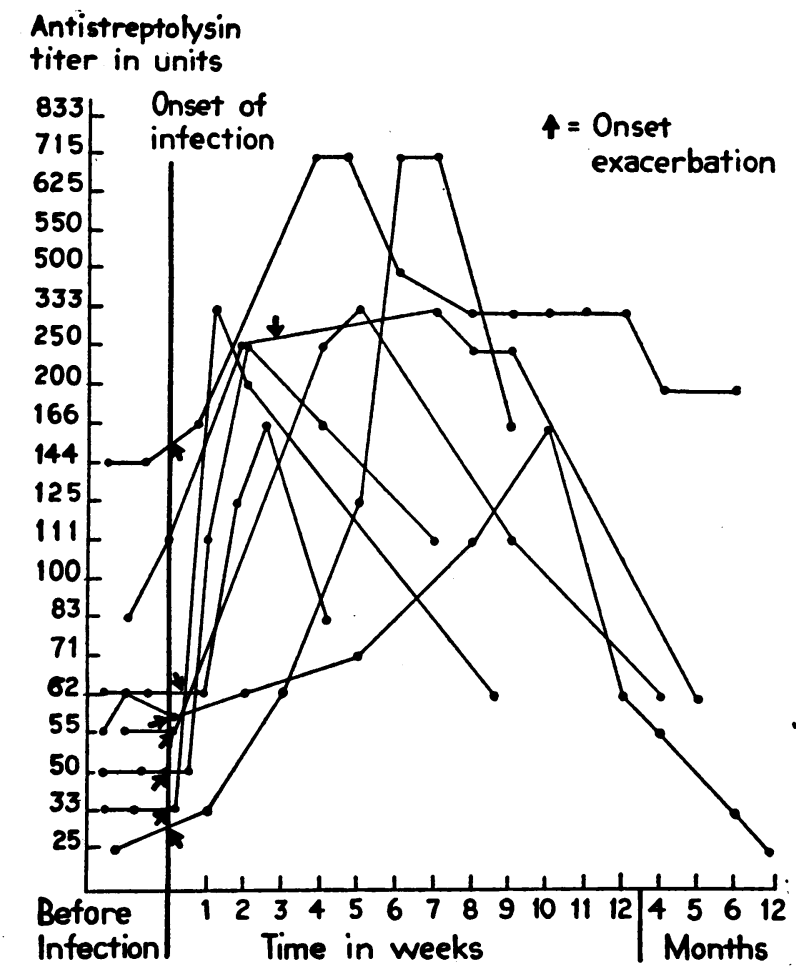

Fig. 3. Relation of Onset of Rise in Antistreptolysin Titer to Onset of Exacerbation in Chronic GLOMERULONEPHRITIS

RELATION OF THE ANTISTREPTOLYSIN TITER RESPONSE TO IMPAIRMENT OF RENAL FUNCTION DURING EXACERBATION IN CHRONIC GLOMERULONEPHRITIS

The renal function was investigated before, during, and after the exacerbations as previously described (1) by means of either the urea clearance test, the phenolsulphonphthalein excretion test, the urea ratio of Mosenthal and Bruger (4), or the serum non-protein nitrogen level, or by a combination of these. As pointed out in the earlier study of exacerbations in chronic glomerulonephritis (1), the inability to demonstrate a decrease in renal function following certain exacerbations may be related either to the slight degree of the renal damage or to failure to employ appropriate renal function tests at sufficiently frequent intervals.

However, some impairment of renal function was demonstrated by one or more of the tests mentioned above in 21 of the 33 exacerbations ${ }^{2}$

2 Protocols of 28 of these exacerbations have been published (1). under consideration in this study. The impairment in function was transient with but one exception (Case $\mathrm{X}, \mathrm{ED}$ ) (1).

The significance of this transient impairment of renal function in the exacerbation in chronic glomerulonephritis is not certain. Goldring $(5,6)$ has shown, using the urea clearance test, that during the acute stage of rheumatic fever and lobar pneumonia there may be renal hyperfunction followed by transient depression of function. Since the exacerbation in chronic glomerulonephritis occurs so soon after the preceding infection, there is the possibility that the transient impairment of renal function observed in many exacerbations in chronic glomerulonephritis may in part represent a peculiar response to infection.

Seventeen of the 24 exacerbations associated with definite rises in antistreptolysin titer showed some evidence of renal function impairment. Similarly, 4 of the 6 exacerbations, in which adequate antistreptolysin titer studies revealed no rises, were associated with impairment of renal function. Thus, the presence or absence of associated rises in titer did not appear to modify the incidence of renal function impairment (as measured in this study) in exacerbations in chronic glomerulonephritis.

\section{DISCUSSION}

As part of a study on the effects of upper respiratory infection on the course of chronic glomerulonephritis, the relation of the serum antistreptolysin titer to the exacerbation in this disease has been investigated. In a previous report (1), it was indicated that each exacerbation observed in chronic glomerulonephritis was preceded by an infection, generally due to the group A hemolytic streptococcus. It was also noted that a transient decrease in renal function was a common feature in exacerbations in chronic glomerulonephritis. In an effort to obtain further data on the mechanism of the development of exacerbations in chronic glomerulonephritis, this analysis of the relation between the serum antistreptolysin titer response and the exacerbation was undertaken.

The data presented above indicate that when patients suffering from chronic glomerulonephritis develop rises in antistreptolysin titer following Group A hemolytic streptococcal infections, the 
incidence of exacerbations is greater among the instances of large rises in titer than among the small rises. This finding does not indicate that a rise in antistreptolysin titer is a prerequisite for the development of an exacerbation (as defined above) in chronic glomerulonephritis, since it has been pointed out that the exacerbation usually occurs before the rise in titer is apparent. More important, 6 instances of exacerbation were not associated with rises in titer. Although the lowest maximum value attained by a rise in antistreptolysin titer associated with an exacerbation in the present study was 144 units, Longcope (7) recorded an instance of exacerbation in which the associated rise in titer reached a maximum of only 100 units. However, it does appear that when there is an antistreptolysin titer response, the higher the rise in titer, the more likely is the occurrence of associated exacerbation.

The explanation for this apparent correlation between the magnitude of the antistreptolysin titer response and the occurrence of exacerbations in chronic glomerulonephritis is not clear from the data at hand. However, several possibilities may be considered, all of which have been mentioned by several authors $(7,8,9,10,11,12,13)$ in discussions of the relation of the serum antistreptolysin titer to various diseases due to or associated with the group A hemolytic streptococcus. For example, certain strains of hemolytic streptococci may be more prone to evoke both exacerbations and larger rises in titer than other strains, although there is no direct evidence to support this possibility.

The possibility that exacerbations follow severe infections rather, than mild ones and that the associated large rises in titer are a reflection of the severity of the preceding infections is not supported by two observations. First, 14 of the 24 exacerbations associated with rises in titer followed mild infections (pharyngitis 12 instances, tonsillitis 1 instance, and "common cold" 1 instance). Indeed, the two highest rises in antistreptolysin titer (1250 and 1666 units) were associated with exacerbations followed infections of this type. And second, as pointed out in the preceding paper (2), there was no apparent correlation between the magnitude of the antistreptolysin titer response in chronic glomerulonephritis and the "deepness" or "superficiality" of the preceding infection. The superficial infections include pharyngitis, tonsillitis and "common cold." Mote and Jones (13) likewise state, “. . . in both the mild and the severe infections every type of antistreptolysin ' $O$ ' curve has been encountered."

The association of exacerbations with large rather than small rises in antistreptolysin titer might be explained by an increase in the ability of chronic nephritics undergoing exacerbation to produce antistreptolysin in response to group A hemolytic streptococcal infections. If this be so, however, it is not clear whether the development of an exacerbation depends on an altered antibody response, whether the reverse is true, or whether both reactions are simply two manifestations of some other process.

There have been some observations that support the view that, in certain diseases due to or associated with group A hemolytic streptococci, the antibody response to the infection differs from the response to uncomplicated infections. Thus, Coburn and Pauli (11) found that attacks of acute tonsillitis and pharyngitis in "normal" individuals were infrequently followed by rises in antistreptolysin titer, and that when rises did occur they rarely reached high values. In contrast, increases in antistreptolysin were more constant and greater in magnitude after similar infections associated with the development of attacks of rheumatic fever. Longcope (7) made similar observations and suggested that, "It might be found that the response of this particular antibody is exaggerated in erysipelas, scarlatina, rheumatic fever and in one form of acute hemorrhagic nephritis." Mote and Jones (13), however, in the summary of their extensive studies on hemolytic streptococcal antibodies in 1336 control cases, 399 cases of hemolytic streptococcal infections, 749 cases of rheumatic fever, and 285 cases of various types of infections, stated: "In our opinion, none of the analyses, including a detailed examination of the antistreptolysin ' $\mathrm{O}$ ' curves in both rheumatic and non-rheumatic subjects, have revealed any basic difference in the hemolytic streptococcal antibodyresponse, insofar as the antibodies investigated are concerned, between the non-rheumatic and the rheumatic individual in their reaction to infection by the hemolytic streptococcus." 
In the present study, although there was a definite correlation between the magnitude of the antistreptolysin titer response (when present) to the incidence of exacerbation in chronic glomerulonephritis, 6 exacerbations were not associated with a rise in titer. Two of these followed hemolytic streptococcal infections and probably represent definite exceptions to the usual association of a rise in antistreptolysin titer, in response to infection, and the development of an exacerbation. However, the other 4 exacerbations, not associated with rises, followed infections not shown to be of hemolytic streptococcal origin. These may possibly have represented some non-specific reaction of the kidneys.

It has been noted that the onset of the exacerbation generally precedes the onset of the rise in serum antistreptolysin titer. This is in striking contrast to the findings of Coburn (14) in regard to rheumatic fever. This author found that attacks of rheumatism usually developed when the titer was still rising, in the second week after the preceding infection. This may represent a real difference between the immune responses of $\mathrm{pa}$ tients with glomerulonephritis and with rheumatic fever, and appears similar to other biological differences between these conditions, discussed in an earlier communication (3).

\section{SUMMARY AND CONCLUSIONS}

1. Thirty-three exacerbations in chronic glomerulonephritis have been observed in 15 of 81 nephritic patients, studied for from 4 months to 8 years. Each exacerbation was preceded by an infection.

2. Twenty-four of these exacerbations were associated with rises in serum antistreptolysin titer, in 6 there was no rise, and in 3 the data were insufficient to determine whether a rise in titer had occurred.

3. When it occurred, the greater the magnitude of the rise in antistreptolysin titer, the greater was the incidence of associated exacerbation in chronic glomerulonephritis.

4. The exacerbation preceded the onset of the rise in antistreptolysin titer in 7 of the 8 sufficiently studied instances.

5. There was a high incidence of transient im- pairment of renal function in exacerbation associated with and also without rises in antistreptolysin titer.

The authors are indebted to Miss Grace Davis and Mr. Walter Meyer for their technical assistance.

\section{BIBLIOGRAPHY}

1. Seegal, D., Lyttle, J. D., Loeb, E. N., Jost, E. L., and Davis, G., On the exacerbation in chronic glomerulonephritis. J. Clin. Invest., 1940, 19, 569.

2. Earle, D. P., Jr., Loeb, E. N., Seegal, D., Lyttle, J. D., and Jost, E. L., The serum antistreptolysin titer in chronic glomerulonephritis. J. Clin. Invest., 1941, 21, 483.

3. Seegal, D., and Earle, D. P., Jr., A consideration of certain biological differences between glomerulonephritis and rheumatic fever. Am. J. M. Sci., 1941, 201, 528.

4. Mosenthal, H. O., and Bruger, M., The urea ratio as a measure of renal function. Arch. Int. Med., $1935,55,411$.

5. Goldring, W., Studies of the kidney in acute infection. II. Observations with the urea clearance test in acute rheumatic infection. J. Clin. Invest., 1931, $10,345$.

6. Goldring, W., Studies of the kidney in acute infection. III. Observations with the urine sediment count (Addis) and the urea clearance test in lobar pneumonia. J. Clin. Invest., 1931, 10, 355.

7. Longcope, W. T., Studies of variations in antistreptolysin titer of blood serum from patients with hemorrhagic nephritis; observations on patients suffering from streptococcal infections, rheumatic fever, and acute and chronic hemorrhagic nephritis. J. Clin. Invest., 1936, 15, 277.

8. Coburn, A. F., and Pauli, R. H., Studies on the relationship of Streptococcus hemolyticus to the rheumatic process. III. Observations on the immunological responses of rheumatic subjects to hemolytic streptococcus. J. Esxper. Med., 1932, 56, 651 .

9. Myers, W. K., and Keefer, C. S., Antistreptolysin content of the blood serum in rheumatic fever and rheumatoid arthritis. J. Clin. Invest., 1934, 13, 155.

10. Wilson, M. G., Wheeler, G. W., and Leask, M. M., The relation of upper respiratory infections to rheumatic fever in children. II. Antihemolysin titers in respiratory infections and their significance in rheumatic fever in children. J. Clin. Invest., 1935, 14, 333.

11. Coburn, A. F., and Pauli, R. H., Studies on the immune response of the rheumatic subject and its relationship to activity of the rheumatic process. VI. The significance of the rise of antistreptolysin level in the development of rheumatic activity. J. Clin. Invest., 1935, 14, 769. 
12. Bunim, J. J., and McEwen, C., The antistreptolysin titer in rheumatic fever, arthritis, and other diseases. J. Clin. Invest., 1940, 19, 75.

13. Mote, J. R., and Jones, T. D., Studies of hemolytic streptococcal antibodies in control groups, rheumatic fever, and rheumatoid arthritis. I. The incidence of antistreptolysin " $O$," antifibrinolysin, and hemolytic streptococcal precipitating antibodies in the sera of urban control groups. II. The frequency of antistreptolysin "O," antifibrinolysin, and precipitating-antibody responses in scąrlet fever, hemolytic streptococcal infections, and rheumatic fever. III. The magnitude of antistreptolysin "O," antifibrinolysin, and precipitating-antibody responses; the persistence of the antibodies, and variations in antistreptolysin " $O$ " curves in scarlet fever, hemolytic streptococcal infections, and rheumatic fever. J. Immunol., 1941, 41, 35.

14. Coburn, A. F., Faulty disposal of Streptococcus hemolyticus in relation to the development of the rheumatic lesion. Tr. and Stud., Coll. Physicians, Philadelphia, 1940, 8, 91. 